This article has been accepted for publication in a future issue of this journal, but has not been fully edited.

Content may change prior to final publication in an issue of the journal. To cite the paper please use the doi propilitudnebsthembigitalahidbrary page.

\section{Estimating fluorescence lifetimes using the expectation-maximization algorithm}

\section{Kai Gao and David Day-Uei Li}

\begin{abstract}
The expectation-maximization algorithm (EM) uses incomplete data to get the estimation of the probabilistic model parameter, and it has been widely used in machine learning. In this paper, EM techniques are applied to estimating fluorescence lifetimes in Time-correlated singlephoton counting based fluorescence lifetime imaging experiments without measuring the instrument response functions. The results of Monte Carlo simulations indicate that the proposed approach can obtain better or comparable accuracy and precision performances than the previously reported method.
\end{abstract}

Introduction: Time-correlated single-photon counting (TCSPC) has excellent timing performances, and it is routinely used for fluorescence lifetime imaging microscopy (FLIM) system [1, 2]. FLIM system is a perfect tool for studying interactions between proteins in living cells [3], and it will have huge potential in the field of medical diagnosis and analysis, and biomedical imaging.

For TCSPC FLIM systems, users usually need to 1) measure the instrument response function (IRF) before conducting FLIM experiments and 2) perform lifetime analysis by solving deconvolution problems $[4,5,6,7]$. However, measuring IRF requires additional efforts, and the experimental setups for measuring IRFs and fluorescence decays are different introducing extra artifacts. Many analysis tools ignore the IRF and use tail-fitting for lifetime analysis [8], but these approaches inevitably introduce errors [5] (especially in recently developed Multi-channel TCSPC systems [8]). In this paper, we will study a new estimation algorithm that can estimate both the IRF and the lifetime for TCSPC FLIM systems, where the IRF is not available or cannot be measured before FLIM experiments.

We have applied the Extended Kalman Filter (EKF) techniques to estimating both the fluorescence lifetime and IRF [9]. EKF can be used to analyze FLIM data when IRF is unknown. However, the photon efficiency of EKF is not comparable with traditional methods.

When a statistical model is closely related to unobservable hidden variables, the maximum a posteriori estimations or the maximum likelihood estimations can be used to estimate model parameters by the expectation-maximization (EM) algorithm. This method is widely used for parameter estimation with incomplete or missing data $[10,11,12]$. Fu et al. used EM to estimate lifetimes [13], but IRF was not involved. In this paper, a new EM-based Lifetime Estimation (EMLE) algorithm is proposed to simultaneously estimate the IRF and lifetime, and it shows better photon efficiency compared with EKF in [9].

Theory: According to the EM theory $[10,11,12]$, we assume that $\left\{x_{i}, i\right.$ $=0,1, \ldots, N-1\}$ are observation values of a random variable $x$ whose density function shown in Fig.1(a) is

$$
f(x \mid \phi)=\sum_{j=0}^{L-1} \lambda_{j} f\left(x \mid \xi_{j}\right),
$$

where $f\left(x \mid \xi_{i}\right)$ is the density function of $i$ th component with a parameter $\xi_{i}$ , $\lambda_{j}$ is the component weight satisfying $\Sigma \lambda_{j}=1, j=0,1, \ldots, L-1$, and $\phi=$ $\left[\xi_{0}, \lambda_{0,}, \xi_{1}, \lambda_{1,}, \xi_{L-1}, \lambda_{L-1}\right]$.

$\xi_{i}$ and $\lambda_{j}$ can be estimated using $\left\{x_{0}, x_{0}, \ldots, x_{N-1}\right\}$. The expectation step (E-step) and the maximization step (M-step) are performed iteratively $[10,11]$, shown in Fig. 1(b).

In E-step, the posterior probability $p_{i j}$ is

$$
p_{i j}=Q_{i}\left(z_{j}\right)=\frac{\lambda_{j} f\left(x_{i} \mid \xi_{j}\right)}{\sum_{j=0}^{L-1} \lambda_{j} f\left(x_{i} \mid \xi_{j}\right)}, i=\mathbf{0}, \mathbf{1}, \cdots, N-\mathbf{1} .
$$

In M-step, $\phi$ that maximizes the expected log-likelihood in E-step can be calculated from

$$
\begin{aligned}
\phi & =\arg \max _{\phi} \sum_{i} \sum_{j} p_{i j} \ln \frac{\lambda_{j} f\left(x_{i} \mid \xi_{j}\right)}{p_{i j}}, \\
& =\arg \max _{\phi} Q(\phi)
\end{aligned}
$$

where $\phi_{i}=\left[\xi_{i}, \lambda_{i}\right]$ is the parameter for the $i$ th component.

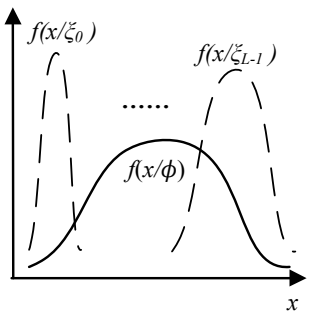

$a$
Fig. 1 Overview of EMLE

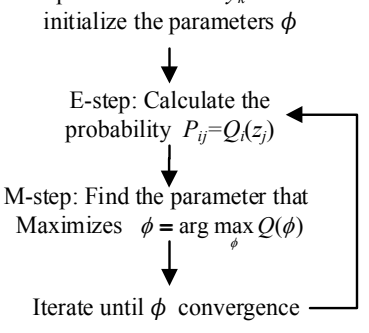

b a Mixture of the density function

$b$ EMLE processing flow.

In a TCSPC-FLIM experiment, we assume that $y(t)$ is the measured decay function, $\operatorname{IRF}(t)$ is the IRF function, and $g(t)$ is the fluorescence density function. $y(t)$ is the sum of an additive Poisson noise, $v(t)$ and the convolution of $\operatorname{IRF}(t)$ and $g(t)[3,7]$

$$
y(t)=g(t) * \operatorname{IRF}(t)+v(t), \quad 0 \leq t \leq T .
$$

We divide the measurement window $T$ into $M$ bins in the TCSPC experiment, and $h=T / M$ is the resolution. Assume that

$$
\begin{aligned}
& g(t)=\frac{1}{\tau} \mathrm{e}^{-\frac{t}{\tau}} \\
& \operatorname{IRF}(t)=\mathbf{0}, \quad t \notin[\mathbf{0}, L h), L h<T
\end{aligned}
$$

According to Eq. (4), after discretizing, the ideal density function is

$$
\begin{aligned}
f_{k} & =g_{k} * I R F_{k}=\frac{\mathbf{1}}{\tau} \mathrm{e}^{-\frac{k h}{\tau}} * I R F_{k} \\
& =\sum_{j=0, i-j \geq 0}^{L-1} \frac{\mathbf{1}}{\tau} \mathrm{e}^{-(i-j) h / \tau} I R F_{j}
\end{aligned}
$$

where $I R F_{j}=I R F(j h), j=0, \ldots, L-1$, and we assume that $\Sigma I R F_{j}=1$.

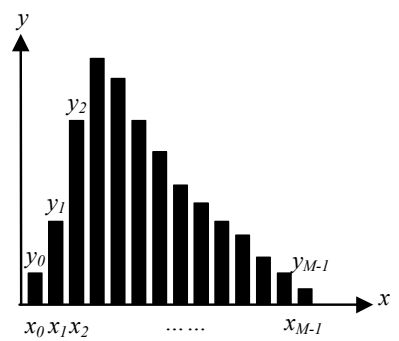

Fig. 2 Histogram measured in TCSPC

In the histogram measured by a TCSPC, $y_{i}=y(i h)$ is photon count in the $i$ th bin $\left(x_{i}=i h\right), i=0,1, \ldots, M-1$, as shown in Fig. 2 , and $\Sigma y_{i}=N_{c} i s$ the total number of all photons. It means that there are in total $N_{c}$ observations, and the histogram is shown in Fig. 2 is the distribution of all observations. So, Eq. (2) can be rewritten as

$$
p_{i j}=\left\{\begin{array}{ccc}
\frac{I R F_{j} \frac{\mathbf{1}}{\tau} \mathrm{e}^{-(i-j) h / \tau} \mid}{\sum_{j=0, i-j \geq \mathbf{0}}^{L-1} \frac{\mathbf{1}}{\tau} \mathrm{e}^{-(i-j) h / \tau} I R F_{j}} & , i \geq j & i=\mathbf{0}, \mathbf{1}, \cdots, M-\mathbf{1} \\
\mathbf{0} & , \text { others }
\end{array} .\right.
$$

Based on Eq. (3), we can obtain

$$
Q(\phi)=\sum_{i=0}^{M-1} \sum_{j=0, i-j-j \mathbf{0}}^{L-1} y_{i} p_{i j} \ln \frac{I R F_{j} \frac{\mathbf{1}}{\tau} e^{-(i-j) h / \tau}}{p_{i j}},
$$

where $\phi=\left[I R F_{0}, I R F_{1}, \ldots, I R F_{L-1}, \tau\right]$.

To deal with the constraint: $\Sigma I R F_{j}=1$, we construct the Lagrangian equations as [14]

$$
\ell(\phi)=Q(\phi)+\beta \sum_{j=0}^{L-1}\left(I R F_{j}-\mathbf{1}\right)
$$


This article has been accepted for publication in a future issue of this journal, but has not been fully edited.

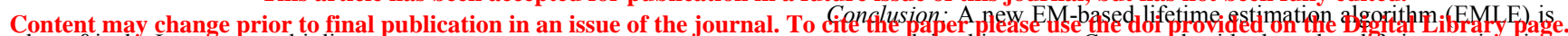
where $\beta$ is the Lagrange multiplier.

Taking the derivative of $\ell(\phi)$ and setting it to be zero, we obtain

$$
\begin{array}{r}
I R F_{j}=\frac{\sum_{i=0}^{M-1} y_{i} p_{i j}}{\sum_{i=0}^{M-1} y_{i}}, \\
\tau=\frac{\sum_{i=0}^{M-1} \sum_{j=0, i-j \geq 0}^{L-1} y_{i} p_{i j}(i-j) h}{\sum_{i=0}^{M-1} \sum_{j=0, i-j \geq 0}^{L-1} y_{i} p_{i j}} .
\end{array}
$$

The initial values of $\phi$ must be assigned firstly before the iterations begin. In this paper, the initial trial values of the $I R F_{j}$ are positive random numbers satisfying $\Sigma I R F_{j}=1$, and the initial lifetime is $\tau=T / 2$.

Monte Carlo Simulations: To analyze the performances of EMLE, Monte Carlo simulations were carried out based on Eq. (4)-(9).

The original IRFs $\left(\operatorname{IRF}_{\mathrm{O}}\right)$ and the estimated IRFs $\left(\operatorname{IRF}_{\mathrm{E}}\right)$ are compared in Fig. 3. Here, IRFs are Poisson density functions, $N_{c}=2048, T=10$ ns, $M=512$, and $L=40$. The full widths at half maximum (FWHM) of IRFs, $\Theta$, are $\Theta_{l}=50 \mathrm{ps}, \Theta_{2}=150 \mathrm{ps}$, and $\Theta_{3}=200 \mathrm{ps}$, respectively. Fig. 3(a) shows $I_{R F}$ and $I_{R F}$ with $\tau=0.5 n s$, and Fig. 3(b) with $\tau=$ $1.5 \mathrm{~ns}$, under different $\Theta \mathrm{s}$. It is obvious that the IRF profiles can be extracted accurately, and the original IRFs and the estimated IRFs agree robustly.

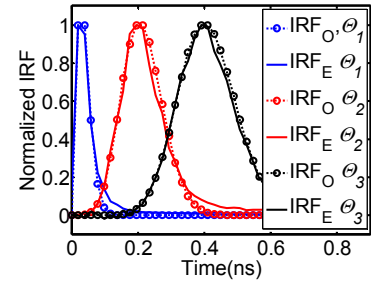

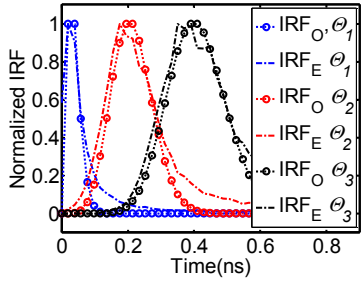

Fig. 3 Comparison of the estimated and original IRFs with different lifetime

a $\tau=0.5 \mathrm{~ns}$

$b \tau=1.5 \mathrm{~ns}$.

Similar to Ref $[9,15]$, the normalized precision of the lifetime estimations, $F$ is defined as $N_{c}^{0.5} \sigma_{\tau} \tau$, and the normalized bias of the lifetime estimations, $E$ is defined as $\left|\tau_{E S T}-\tau\right| / \tau$.

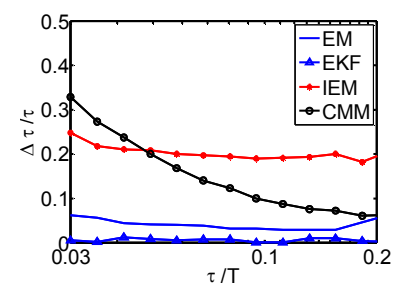

$a$

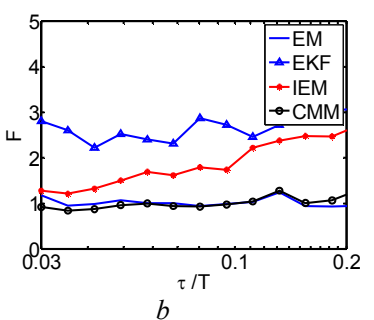

$b$

Fig. 4 Comparison of bias and precision $a E$ $b F$.

Monte Carlo simulations were performed to evaluate the precision and accuracy of lifetime estimations. Fig. 4 shows the results obtained from EMLE and other previously reported algorithms. Here, $\Theta=150 \mathrm{ps}$ for all, $M=1024$ for CMM [16], $M=1024$ for EMLE and EKF, $M=15$ for IEM [7]. T, $L, N_{c}$ for Fig. 4 are the same as above, and all parameters for EKF are the same as those in [9]. From Fig. 4, the bias performances of EMLE $(E<0.05)$ is slightly worse than that of EKF when $0.04<\tau / T<0.2$. Large biases were produced by both CMM and IEM showing that the IRF calibration before conducting FLIM experiments is essential, whereas the photon efficiency of EMLE is comparable to CMM with the $F$-value $\sim 1$, and the bias produced by EMLE is negligible.

proposed in this paper. Compared with the other lifetime estimation approaches such as CMM and IEM, EMLE does not require IRF calibration before conducting FLIM experiments, and it can estimate IRF and the lifetime simultaneously. Monte Carlo Simulations confirm that the proposed EMLE can correctly estimate the lifetime, and it has better photon efficiency than the EKF approaches when $0.04<\tau / T<$ 0.2 .

Kai Gao and David Day-Uei Li (Centre for Biophotonics, University of Strathclyde, Glasgow, United Kingdom)

E-Mail: gaokai000@hotmail.com

Kai Gao: Also with School of Electronic Science, National University of Defense Technology, Changsha, People's Republic of China

\section{References}

1. Turgeman, L. and D. Fixler: 'Photon efficiency optimization in time correlated single photon counting technique for fluorescence lifetime imaging systems', IEEE Trans. Biomed. Eng., 2013. 60(6), pp. 15711579, doi: 10.1109/TBME.2013.2238671

2. Okabe, K., Inada, N., Gota, C., Harada, Y., Funatsu, T., and Uchiyama, S.: 'Intracellular Temperature Mapping with a Fluorescent Polymeric Thermometer and Fluorescence Lifetime Imaging Microscopy', Nature Communications, 2012, 3, pp. 705, doi: 10.1038/ncomms 1714

3. Becker, W.: 'Advanced time-correlated single photon counting applications' (Springer International Publishing, 2015).

4. PicoQuant: 'Lifetime fitting using the film script step by step', http://picoquant.com/images/uploads/downloads/lifetime-

fitting_using_the_flim-script_step_by_step.pdf, accessed 2013

5. Zhang, Y., Cuyt, A., Lee, W.S., et al: 'Towards unsupervised fluorescence lifetime imaging using low dimensional variable projection', Opt. Express, 2016. 24(23), pp.26777-26791, doi: 10.1364/OE.24.026777.

6. Jo, J.A., Fang, Q., Papaioannou, T., and Marcu, L.: 'Fast model-free deconvolution of fluorescence decay for analysis of biological systems', J. Biomed. Opt. 2004. 9(4), pp.743-752, doi:10.1117/1.1752919

7. Liu, J., Sun, Y., Qi, J., and Marcu, L.: 'Novel method for fast and robust estimation of fluorescence decay dynamics using constrained least-squares deconvolution with Laguerre expansion', Phys. Med. Biol. 2012. 57(4), pp. 843- 865, doi: 10.1088/0031-9155/57/4/843

8. Li, D.-U., Arlt, J., Richardson, J., et al: 'Real-time fluorescence lifetime imaging system with a $32 \times 320.13$ microm CMOS low darkcount single-photon avalanche diode array', Opt Express, 2010. 18(10), pp.10257-69. doi: 10.1364/OE.18.010257

9. Gao, K., Li, D. D.-U., 'Estimating fluorescence lifetimes using extended Kalman filter', Electronics Letter, 2017. 53(15), pp.10271029, doi: 10.1049/el.2017.1085

10. Bordes, L., Chauveau, D.: 'EM and stochastic EM algorithms for reliability mixture models under random censoring'. Computational Statistics, 2013. 31(4), pp 1513-1538, doi:10.1007/s00180-016-0661-7

11. Byrne, C., Eggermont P. P.B.: 'Handbook of mathematical methods in imaging' (New York: Springer, 2015, 2nd Edition)

12. Koch, K.R.: 'Expectation Maximization algorithm and its minimal detectable outliers', Studia Geophysica et Geodaetica. 2017. 61(1). pp.1-18, doi:10.1007/s11200-016-0617-y

13. Fu, C. Y., Ng, B.-K., Razul, S. G.: 'Fluorescence lifetime discrimination using expectation-maximization algorithm with join deconvolution', J. Biomed. Opt, 2009. 14(6), pp. 064009, doi.org/10.1117/1.3258835

14. Sheng, Y.H.: 'Practical mathematics handbook' (Beijing: Springer, Science press, 1992)

15. Gerritsen, H. C., Asselbergs, M. A. H., Agronskaia, A. V., et al: 'Fluorescence lifetime imaging in scanning microscopes: acquisition speed, photon economy, and lifetime resolution', Journal of Microscopy, 2002. 206(3), pp. 218-224, doi:10.1046/j.13652818.2002.01031.x

16. Li, D. D.-U., Arlt, J., Tyndall, D., Walker, R., et al: 'Video-rate fluorescence lifetime imaging camera with CMOS single-photon avalanche diode arrays and high-speed imaging algorithm', J. Biomed. Opt, 2011. 16(9), pp. 096012, doi.org/10.1117/1.3625288 